\title{
An Introduction to Rocker: Docker Containers for $\mathbf{R}$
}

by Carl Boettiger, Dirk Eddelbuettel

\begin{abstract}
We describe the Rocker project, which provides a widely-used suite of Docker images with customized R environments for particular tasks. We discuss how this suite is organized, and how these tools can increase portability, scaling, reproducibility, and convenience of R users and developers.
\end{abstract}

\section{Introduction}

The Rocker project was launched in October 2014 as a collaboration between the authors to provide high-quality Docker images containing the R environment (Boettiger and Eddelbuettel, 2014). Since that time, the project has seen both considerable uptake in the community and substantial development and evolution. Here we seek to document the project's objectives and uses.

\section{What is Docker?}

Docker is a popular open-source tool to create, distribute, deploy, and run software applications using containers. Containers provide a virtual environment (see Clark et al. (2014) for an overview of common virtual environments) requiring all operating-system components an application needs to run. Docker containers are lightweight as they share the operating system kernel, start instantly using a layered filesystem which minimizes disk footprint and download time, are built on open standards that run on all major platforms (Linux, Mac, Windows), and provide an added layer of security by running an application in an isolated environment (Docker, 2015). Familiarity with a few key terms is helpful in understanding this paper. The term "container" refers to an isolated software environment on a computer. $\mathrm{R}$ users can think of running a container as analogous to loading an $\mathrm{R}$ package; a container is an active instance of a static Docker image. A Docker "image" is a binary archive of that software, analogous to an R binary package: a given version is downloaded only once, and can then be "run" to create a container whenever it is needed. A "Dockerfile" is a recipe, the source-code, to create a Docker image. Pre-built Docker images are publicly available through Docker Hub, which plays a role for central distribution similar to CRAN in our analogy. Development and contributions to the Rocker project focus on the construction, organization and maintenance of these Dockerfiles.

\section{Design principles and use cases}

Docker gives users very convenient access to pre-configured and pre-built binary images that "just work". This allows $\mathrm{R}$ users to access a wider-variety of ready-to-use environments than provided by either the R Project itself or, say, their distribution which will generally focus on one (current) release. For example, R users on Windows may run RStudio ${ }^{\circledR}$ Server or Shiny ${ }^{\circledR}$ Server locally just by launching a single command (once Docker itself is installed). Another common use-case is access to R-devel without affecting the local system. Here, we detail some of the principal use cases motivating these containerized versions of $\mathrm{R}$ environments, and the design principles that help make them work.

\section{Portability: From laptop to cloud}

One common use case for Rocker containers is to provide a fast and reliable mechanism to deploy a custom R environment to a remote server, such as Amazon Web Services Elastic Compute (AWS EC2), DigitalOcean, NSF's Jetstream servers (Stewart et al., 2015), or private or institutional server hardware. Rocker containers are also easy to run locally on most modern laptops using Windows, MacOS, or Linux-based operating systems. By sharing volumes with the local host, users can still manipulate files with familiar, native tools while performing computation through a reproducible, containerized environhment (Boettiger, 2015). Being able to test code in a predictable, pre-configured R environment on a local machine and to then run the same code in an identical environment on a remote server (e.g., for access to greater RAM, more processors, or merely to free up the local machine from a long-running computation) is essential for low-friction scaling of analysis. Without such containerization, getting code to run appropriately in a remote environment can be a major undertaking, requiring both time and knowledge many would-be users may not have. 
For instance, on any platform with Docker installed, the following Docker command will launch a Rocker container providing the RStudio ${ }^{\circledR}$ server environment over a web interface.

wget -q0- https://get.docker.com/ I sh

sudo docker run -p 8787:8787 -e PASSWORD=<PICK-A-PASSWORD> rocker/rstudio

The docker run option $-p$ sets the port on which RStudio ${ }^{\circledR}$ will appear, for which 8787 is the default (adding your user to the docker group avoids the need for a sudo command to call docker: sudo usermod -g docker \$USER). Many academic and commercial cloud providers make it possible to execute such code snippets when a container is launched, without ever needing to ssh into the machine. The user may log into the server merely by pasting its IP address or DNS name (followed by the chosen port, e.g., : 8787) into a browser and entering the appropriate password. This provides the user with a familiar, interactive environment running on a remote machine while requiring a minimum of expertise.

This portability is also valuable in an instructional context. Requiring students to install all necessary software on personal laptops can be particularly challenging for short workshops, where download and installation time and troubleshooting across heterogeneous machines can prove time consuming and frustrating for students and instructors alike. By deploying a Rocker image or Rockerderived image (see Extensibility) on a cloud machine, an instructor can easily provide all students access to the pre-configured software environment using only the browser on their laptops. This strategy has proven effective in our own experience in both workshops and semester-length courses. Similar Docker-based cloud deployments have been scaled to courses of 100s of students, e.g., at Duke (Cetinkaya-Rundel and Rundel, 2017) and UC Berkeley (UC Berkeley, 2017).

\section{HPC application}

The portability of Rocker images can be particularly valuable in High Performance Computing contexts Setting up a specific R environment on High Performance Computing platforms and other centrally administrated multi-user machines or clusters has traditionally been challenging due to restrictions on root access that may be needed to install certain libraries. Versions of $R$ and packages installed by the system administrator may also lag behind the most recent releases. Deploying Docker containers on HPC systems has previously been more very problematic since most system administrators do not want to allow the elevated user permissions the Docker runtime environment requires. To work around this problem, Lawrence Berkeley National Labs (LBNL) has made 'Singularity' (Lawrence Berkeley National Laboratories, 2017): a container runtime environment that users can both install and use to run most Docker containers without requiring root privileges. Singularity has seen rapid adoption in the HPC community (http://singularity. lbl.gov/install-request). Rocker containers can be run through Singularity with a single command much like the native Docker commands, e.g.

singularity exec docker://rocker/tidyverse:latest $\mathrm{R}$

More details can be found in the Singularity documentation.

\section{Interfaces}

An important aspect of the Rocker project design is the ability for users to interact with the software on the container through either an interactive shell session (such as the R shell or a bash shell), or through a web browser accessing the RStudio ${ }^{\circledR}$ Server integrated development environment (IDE). Traditional remote and high-performance computing workflows for $\mathrm{R}$ users have usually required the use of ssh and a terminal-only interface, posing a challenge for interactive graphics and a barrier to users unfamiliar with these tools and environments. Accessing an RStudio ${ }^{\circledR}$ container through the browser removes these barriers. Rocker images include the RStudio-server software pre-installed and configured with the explicit permission of RStudio ${ }^{\circledR}$ Inc.

Users can access a bash shell running as root within a Rocker container using

docker exec -ti <container-id> bash

which can be useful for administrative tasks such as installing system dependencies. All Rocker images can also be run as an interactive R, RScript or bash shell without running RStudio, which can be useful for batch jobs or for anyone who prefers that environment.

As with any interactive Docker container, users should specify the terminal $(-t)$ and interactive (-i) flags, (here combined with interactive as $-t i)$, and specify the desired executable environment (e.g., $\mathrm{R}$, though other common options could be Rscript or bash): 
docker run --rm -ti rocker/tidyverse $\mathrm{R}$

This example shows the use of the --rm flag to indicate that the container should be removed when the interactive session is finished. Details on sharing volumes, managing user permissions, and more can be found on the Rocker website, https://rocker-project.org.

\section{Sandboxed}

Another feature of Rocker containers is the ability to provide a sandboxed environment, isolated from software and potentially from other data on the machine. Many users are reluctant to upgrade their suite of installed packages, which may break their existing code or even their R environment if the installation goes poorly. However, upgrading packages and/or the R environment is often necessary to run analyses from a colleague, or access more recent methods. Rocker offers an easy solution. For instance, a user can run $\mathrm{R}$ code requiring the most recent versions of $\mathrm{R}$ and related packages inside a Rocker container without having to upgrade their local installations first. Conversely, one could use Rocker to run code on an older $\mathrm{R}$ release with prior versions of $\mathrm{R}$ packages, again without having to make any alteration to one's local $\mathrm{R}$ install. Another common use case is to access a container with support for particular options such as using gcc or clang compiler sanitizers (Eddelbuettel, 2014). These require $R$ itself be built with specialized settings that may not be not available or familiar to many $\mathrm{R}$ users on their native system, but can be easily deployed by pulling the Rocker images rocker/r-devel-san or rocker/r-devel-ubsan-clang.

This sandboxing feature is also valuable in the remote computing context, allowing system administrators to grant users freedom to install software which requires root privileges inside a container, while not granting them root access on the host machine. Root access is required to launch Docker containers, though not to access containers already running and providing some service such as RStudio. Users logging into a container through the RStudio ${ }^{\circledR}$ interface do not by default have root privileges, though are able to install $\mathrm{R}$ packages. Granting these users root privileges in the container still leaves them sandboxed from the host container. Sandboxing also serves an important function in reproducible research by making it easier to test a specified environment in isolation from the host machine. Unlike traditional virtual machines, containers do not impose a large footprint of reserved resources as a typical host can easily support 100s of containers (Docker, 2015).

\section{Transparent}

Users can easily determine the software stack installed on any Rocker image by examining the associated Dockerfile recipe, which provides a concise, human-readable record of the installation. All Rocker images use automated builds through Docker Hub, which also acts as the central, default repository distributing the images. Using automated builds rather than uploading pre-built image binaries to Docker Hub avoids the potential for the build not to match the recipe. The corresponding Dockerfile is visible both on the Docker Hub and in the linked GitHub repository, which provides a transparent versioned history of all changes made to these recipes, as well as documentation, a community wiki, and issue trackers for discussing proposed changes, bugs, improvements to the Dockerfiles and troubleshoot any issues users may encounter. Having these public source files built automatically by a trusted provider (Docker Hub), rather than built locally and uploaded as binaries, is also useful from a security perspective in avoiding malware.

\section{Community optimized}

Having a shared, transparent computational environment created by a publicly hosted, reproducible recipe facilitates community input into configuration details. $\mathrm{R}$ and many of its packages and related software can be configured with a wide range of options, compilers, different linear-algebra libraries and so forth. While this flexibility reflects varying needs, many users rely on default settings which are most often are optimized more for simplicity of installation rather than than performance. The Rocker recipes reflect significant community input on these choices. This helps create a more finely tuned, optimized reference implementation of the R environment as well as a platform for comparing and discussing these concerns which are often overlooked elsewhere. Issues and Pull Requests on the Rocker repositories on GitHub attest to some of these discussions and improvements. In particular, input from the Docker Inc. employees through the official approval process for the $r$-base image, expertise from the Debian R maintainer and other Debian developers, and both direct and indirect feedback from the experience and user-generated documentation from many early adopters in the $\mathrm{R}$ community has helped shape and strengthen the project over the past few years. Widespread use of the Rocker image helps promote both testing of these choices and contributions, further tweaking the configuration from many members of the R community. 


\section{Versioned}

Access to specific versions of software can be important for users who need computational reproducibility more than having the latest release of any piece of software, since subsequent releases can alter the behavior of code, introduce errors or otherwise alter previous results. The versioned stack ( $r$-ver, rstudio, tidyverse, verse, and geospatial) provides images which are intended to build an identical software stack every time, regardless of the release of new libraries and packages. Users should specify an $\mathrm{R}$ version tag in the Docker image name to request a version stable image, e.g., rocker/verse: 3.4.0. If no tag is explicitly requested, Docker will provide the image with the tag : latest, which will always have the latest available versions of the software (built nightly).

Users building on the version-tagged images will by default use the MRAN snapshot mirror (Revolution Analytics, 2017) associated with the most recent date for which that image was current. This ensures that a Dockerfile building FROM rocker/verse:3.4.1 will only install R package versions that were available on CRAN on 2017-06-30, i.e., the day R 3.4.1 was released. This default can of course be overwritten in the standard $\mathrm{R}$ manner, e.g., by specifying a different CRAN mirror explicitly in any command to install packages, e.g., install. packages(), or by adjusting the default CRAN mirror in options (repo=<CRAN-MIRROR $>$ ) in an . Rprofile. Note that the MRAN date associated with the current release (e.g., 3.4.2 at the time of writing) will continue to advance on the Docker-hub image until the next $\mathrm{R}$ release. Software installed from apt-get in these images will come from the the stable Debian release (stretch or jessie) and thus not change versions (though it will receive security patches). Packages installed from BioConductor using the bioclite() utility will also install the version appropriate to the version of $\mathrm{R}$ found on the system (the Bioconductor semi-annual release model avoids the need for an MRAN mirror). Users installing packages from GitHub or other sources can request a specific git release tag or hash for a more reproducible build, or adopt an alternative approach such as packrat (Ushey et al., 2016). A more general discussion of the use and limitations of Docker for computational reproducibility can be found in Boettiger (2015).

\section{Extensible}

Any portable computational environment faces an inevitable tension between the "kitchen sink problem" at one extreme, and the "discovery problem" on the other. A kitchen sink image seeks to accommodate too many use cases in a single image. Such images are inevitably very large and thus slow or difficult to deploy, maintain and optimize. At the other extreme, providing too many specialized images makes it more difficult for a user to discover the one they need. The Rocker project seeks to avoid both of these problems by providing a carefully-curated suite of images that an be easily extended by individuals and communities.

To make extensions transparent and persistent, Rocker images can be extended by any user by writing their own Dockerfiles based on an appropriate Rocker image. The Dockerfiles in the Rocker stack should themselves provide a simple example of this, (as described in the following section). A user begins by selecting an appropriate base image for their needs: if the RStudio ${ }^{\circledR}$ interface is desired, a user might start with FROM rocker/rstudio; an image for testing an R package with compiled code might use FROM rocker/r-devel-san, and an image for reproducing a data analysis will probably select a stable version tag in addition to an appropriate base library, e.g.,: FROM rocker/tidyverse:3.4.1. Users can easily add additional software to any running Rocker image using the standard R and Debian mechanisms. Details on how to extend Rocker images can be found at https://rocker-project.org.

Sharing these Dockerfiles can also facilitate the emergence of extensions tuned to particular communities. For instance, the rocker/geospatial image emerged from the input of a number of Rocker users all adding common geospatial libraries and packages on top of the existing Rocker images. This coalescence helped create a more fine-tuned image with broad support for a wide range of commonly-used data formats and libraries. Other community images are developed and maintained independently of the Rocker project, such as the popgen image of population-geneticsoriented software developed by the National Evolutionary Synthesis Center (NESCent). Rocker images are also being used as base Docker images in the NSF sponsored Whole Tale project for reproducible computing (Ludaescher et al., 2017), and are heavily used by the rhub project in automated package testing (Csárdi, 2017).

\section{Rocker organization and workflow}

The Rocker project consists of a suite of images built automatically by and hosted on the Docker Hub, https://hub. docker.com/r/rocker. Source Dockerfiles, supporting scripts and documentation are hosted on GitHub under the organization rocker-org, https://github.com/rocker-org. The 
issue tracker and pull requests are used for community input, discussions, and contributions to these images. The Rocker project wiki, https://github. com/rocker-org/rocker/wiki, provides a place to synthesize community-contributed documentation, use-cases, and other knowledge about using the Rocker images.

\section{Images in the Rocker Project}

The Rocker project aims to provide a small core of Docker images that serve as convenient 'base' images on which other users can build custom R environments by writing their own Dockerfiles, while also providing a 'batteries included' approach to images that can be used out of the box. The challenges of balancing diverse needs driven by very different use cases against the overarching goals of creating images that are still sufficiently light-weight, easy to use, and easy to maintain is a difficult art. The implementation in both individual Rocker images and image stacks can never perfect that balance for everyone, but today reflects the considerable community input and testing over the past few years.

All Rocker images are based on the Debian Linux distribution. It provides a small base image, the well-known apt package management system, and a rich ecosystem of software libraries, making it the base image of choice for Docker images, including many of the "official" images maintained by Docker's own development team. The Debian platform is also perhaps the best-supported Linux platform within the R community, including an active $r$-sig-debian listserve. The relatively long period between stable Debian releases (roughly two years recently) means that software in the Debian stable (e.g., debian: jessie, debian: stretch) releases can lag significantly behind current releases of popular software, including R. More recent versions of packages can be found in the pre-release distribution, debian: testing, while the very latest binary builds can be found on debian: unstable. The Rocker project can be largely divided into two stacks which address different needs, reflected in which Debian distribution they are based on. The first stack is based on debian: testing. The second, more recently-introduced stack, is based only on Debian stable releases. Rocker images always point to specific stable releases (jessie, stretch), and do not use the tag debian: stable, which is a rolling tag that always points to the most recent stable version. The different Rocker stacks have different aims and thus provide different images, as shown in Tables $1 \& 2$ below.

\section{The debian: testing-based images}

The debian: testing stack aims to make the most efficient use of upstream builds: the pre-compiled . deb binaries provided by the Debian repositories. It is both quicker and easier to install software from binaries, since the package manager (apt) manages the necessary (binary) dependencies and bypasses the time-consuming process of compiling from source. Basing this stack on debian: testing means that much more recent versions of commonly-used libraries and compilers are available as binaries than would be found in a Debian stable release. In order to provide optional access to the most recent available binaries, this stack uses apt-pinning (Debian Project, 2017) to allow the apt package manager to selectively install binaries from debian: unstable, which represents the most recent set of packages built for Debian. Similarly, recent versions of many popular R packages can also be installed pre-built through the package manager, e.g., apt-get install $r$-cran-xml. This can be particularly helpful for packages with external system dependencies (such as libxml2-dev in this example) which cannot be installed from the $\mathrm{R}$ console as they are system dependencies rather than $\mathrm{R}$ packages installed from within R. We should note, however, that only about 500 of the over 11,000 CRAN packages are available as Debian packages.

As the names testing and unstable imply, particular versions of package can change as packages move from unstable into testing. New versions are sent to unstable during the normal course of Debian development. This can occasionally break a previously-working installation command in a Dockerfile until the maintainer redirects the package manager to install a package from the unstable sources that could previously be installed from testing, or vice versa (using the $-t$ option in apt). That said, packages only migrate from unstable to testing after a period of several days-and if the migration and installation of the particular version is free of interactions with other packages in their dependency graph. That way, unstable serves as validation lab which leaves testing reasonably stable yet current.

Relative to stable, the testing stack thus offers some advantages as almost all software can be installed through the package manager. Installation of binary packages from testing generally provides the most recent available software, and installs it quickly as a binary. On the other hand, these Dockerfiles may require occasional maintenance when packages migrate and/or versions change. The resulting images are also inherently dynamic: rebuilding the same Dockerfile months or years apart will generate images with significantly different versions of software installed as the pool of 
underlying packages changes through time.

\section{Images overview}

The debian: testing-based stack currently includes seven images actively maintained by the Rocker development team (Table 1). $r$-base builds on debian: testing, and the other six in the stack each build directly from $r$-base. The $r$-base image is unique in that it is designated as the official image for the $\mathrm{R}$ language by the Docker organization itself. This official image is reviewed and then built by employees of Docker Inc. based on a Dockerfile maintained by the Rocker team. Consequently, users should refer to this image in Docker commands without an organization namespace, e.g., docker run -ti $r$-base to access the official image. All other images in the Rocker project are not individually reviewed and built by Docker Inc. and must be referenced using the rocker namespace, e.g., docker run -ti rocker/r-devel.

Several of the images in this stack are oriented towards the R development community: $r$-devel, drd, $r$-devel-san, and $r$-devel-ubsan-clang which all add a copy of the development version of $\mathrm{R}$ side-by-side to the current release of $\mathrm{R}$ provided by $\mathrm{r}$-base. On these images, the development version is aliased to RD to distinguish from the current release, $R$. As the names suggest, each provide slightly different configurations. Of particular interest are the images providing development $\mathrm{R}$ built with support for $\mathrm{C} / \mathrm{C}++$ address and undefined-behavior sanitizers, which are somewhat difficult to configure (Eddelbuettel, 2014).

As these images focus on developers and/or as base images for custom uses, this stack does not include many specific R packages. Additional dependencies and packages can easily be installed from apt. $R$ packages not available in the apt repositories can be installed directly from CRAN using either $\mathrm{R}$ or the littler scripts, as described in https://rocker-project.org/use.

This stack also includes the images shiny and rstudio: testing that provide Shiny server and RStudio $^{\circledR}$ server IDE from RStudio ${ }^{\circledR}$ Inc, built on the $r$-base image. RStudio ${ }^{\circledR}$ and Shiny are registered trademarks of RStudio Inc, and their use and the distribution of their software in binary form on Docker Hub has been granted to the Rocker project by explicit permission from RStudio. Users should review RStudio ${ }^{\circledR}$ 's trademark use policy (http: // www. rstudio. com/about/trademark/) and address inquiries about further distribution or other questions to permissions@rstudio.com. The Rocker project also provides images with RStudio ${ }^{\circledR}$ server and Shiny server in the stable versioned stack.

Build schedule: The official $r$-base image is rebuilt by Docker following any updates to the official debian images (roughly every few weeks). The rest of the stack uses build triggers that rebuild the images whenever $r$-base is updated or the Dockerfile sources are updated on the corresponding GitHub repository. The only exception in this stack is the drd image, which is rebuilt each week by a cron trigger.

Table 1: The debian: testing image stack

\begin{tabular}{llll}
\hline image & description & size & downloads \\
\hline r-base & official image with current version of R & $254 \mathrm{MB}$ & 632,000 \\
r-devel & R-devel added side-by-side to r-base (using alias & $1 \mathrm{~GB}$ & 4,000 \\
& RD) & $571 \mathrm{MB}$ & 4,000 \\
drd & lightweight r-devel, built weekly & $1.1 \mathrm{~GB}$ & 1,000 \\
r-devel-san & as r-devel, but built with compiler sanitizers & $1.1 \mathrm{~GB}$ & 525 \\
r-devel-ubsan-clang & sanitizers, clang c compiler (instead of gcc) & $1.1 \mathrm{~GB}$ & 1,000 \\
rstudio:testing & rstudio on debian:testing & $409 \mathrm{MB}$ & 123,000 \\
shiny & shiny-server on r-base & & \\
\hline
\end{tabular}

Table 2: The rocker-versioned stack of images

\begin{tabular}{llll}
\hline image & description & size & downloads \\
\hline r-ver & version-stable base R \& src build tools & $219 \mathrm{MB}$ & 6,000 \\
rstudio & adds rstudio & $334 \mathrm{MB}$ & 314,000 \\
tidyverse & adds tidyverse \& devtools & $656 \mathrm{MB}$ & $83,000^{1}$ \\
verse & adds java, tex \& publishing-related packages & $947 \mathrm{MB}$ & 9,000 \\
geospatial & adds geospatial libraries & $1.3 \mathrm{~GB}$ & 4,000 \\
\hline
\end{tabular}

\footnotetext{
${ }^{1}$ This figure includes 49,000 downloads under the earlier name hadleyverse.
} 


\section{The debian: stable-based stack}

This stack emphasizes stability and reproducibility of the Docker build. This stack was introduced much more recently (November 2016) in response to considerable user input and requests. The key feature of this stack is the ability to run older versions of $\mathrm{R}$ along with the then-contemporaneous versions of $\mathrm{R}$ packages. A user specifies the version desired using an image tag, e.g., rocker $/ r$-ver: 3.3 .1 will refer to an image with $R$ version 3.3.1 installed. Omitting the tag is equivalent to using the tag latest, which, as the name implies, will always point to an image using the current $\mathrm{R}$ release. Thus, users who want to create downstream Dockerfiles, which are based on the current release at the time (but will continue to reconstruct the same environment in the future after newer $\mathrm{R}$ versions are released), should explicitly include the corresponding version tag, e.g., rocker/r-ver:3.4.2 at the time of writing, and not the latest tag. Users can also run the current development version of $\mathrm{R}$ using the tag devel, which is built nightly from R-devel sources from subversion.

MRAN archives: To facilitate installation of only contemporaneous versions of $R$ packages on these images, the default CRAN mirror from which to install $R$ packages is fixed to a snapshot of CRAN corresponding to the last date for which that version of $\mathrm{R}$ was current (e.g., 3.4 . 2 was released on 201709-28, thus 3.4. 1 is pinned to the MRAN snapshot for that date). These snapshots are provided by the MRAN archive created by Revolution Analytics (now part of Microsoft). It archives daily snapshots of all of CRAN from which a user can install packages with the usual install. packages() function (Revolution Analytics, 2017). Users can always override this default by passing any current CRAN repository explicitly. Unlike CRAN, Bioconductor only updates its repositories through bi-annual releases aligned to R's spring release schedule. Thus, Bioconductor packages can be installed in the usual way using bioclite, which automatically selects the Bioconductor release corresponding to the version of $R$ in use.

Version tags: The version tags are propagated throughout this stack: e.g., rocker/tidyverse : devel will provide the currently-released versions of the R packages in the tidyverse (Wickham, 2017) installed on the nightly build of R-devel. Developers building packages on this stack are encouraged to tag their images accordingly as well. Table 3 indicates which versions of $\mathrm{R}$ are currently available in the stack, going back to 3.1.0. While older versions may be added to the stack at a later date, we note that the MRAN snapshots began in 2014-09-17 and thus go back only to the R 3.1 era. Each tag must be built from a separate Dockerfile, enabling minor differences in the build instructions to accommodate changing dependencies. Dockerfiles for past versions (e.g., prior to 3.4.2 currently) are intended to remain static over the long term, while the tag for the current version, latest, and devel may be tweaked to accommodate new features or dependencies. Version tags also obey semantics so that omitting the second or third position of the tag is identical to asking for the most recent version: i.e., rocker/verse:3.3 is the same as rocker/verse:3.3.3, and rocker/verse: 3 is (at the time of writing), rocker/verse: 3.4.2. This is accomplished using post-build hooks in Docker Hub-see examples at https://github.com/rocker-org/rocker-versioned/for details.

Installation: In this stack, the desired version of $\mathrm{R}$ is always built directly from source rather than the apt repositories. Compilers and dependencies are still installed from the stable apt repositories, and thus lag behind the more recent versions found in the testing stack. Version tags 3.3.3 and older are based on the Debian 8.0 release, code-named jessie, while 3.4.0-3.4.2, devel, and latest are based on Debian 9.0, stretch, (released 2017-06-17, while R was at 3.4.0), and thus have access to much newer versions of common system dependencies and compilers. Dependencies needed to compile $\mathrm{R}$ that are not required at runtime are removed once $\mathrm{R}$ is installed, keeping the base images light-weight for faster download times. While most system dependencies required by common $\mathrm{R}$ packages can still be installed from the apt repositories, occasionally a more recent version must be compiled from source (e.g., the Gibbs Sampling program JAGS (Plummer, 2017), and the geospatial toolkit GDAL, must both be compiled from source on debian: jessie images). In this stack, users should avoid installing $R$ packages using apt without careful consideration as this will install a second (probably different) version of $\mathrm{R}$ from the Debian repositories, and a dated version of the $\mathrm{R}$ package since any $r$-cran-pkgname package in the Debian repositories will depend on $r$-base in apt as well.

Build schedule: All images are built automatically from their corresponding Dockerfiles (found in the GitHub repositories rocker-org/rocker-versioned and rocker-org/geospatial). A cron job sends nightly build triggers to Docker Hub to rebuild the latest and devel tagged images throughout the stack. To decrease load on the hub, build triggers for the numeric version tags are sent monthly. Although the Dockerfiles for older $\mathrm{R}$ versions install an almost-identical software environment every time, the monthly rebuilding of these images on Docker Hub ensures they continue to receive Debian security updates from upstream, and proves the build recipe still executes successfully. Note that rebuilding images with software from external repositories never produces a bit-wise identical image, and thus the image identifier hash will change at each build. 


\section{Images overview}

In this stack, each image builds on the previous image, rather than all other images building directly on the base image, as in the testing stack. Table 2 lists the names and descriptions of the five images in this stack, along with image size and approximate download counts from Docker Hub. Sizes reflect (compressed) cumulative size: a user who has already downloaded the most recent version of $r$-ver and then pulls a copy of rstudio image will only need to download the additional $115 \mathrm{MB}$ in the rstudio layers and not the full $334 \mathrm{MB}$ listed. This linear design limits flexibility (no option for tidyverse without rstudio) but simplifies use and maintenance. While no single environment will be optimal for everyone, both the packages selected in this stack and the stack ordering reflect considerable community input and tuning.

The rstudio image includes a lightweight, easy-to-use and docker-friendly init system, s6 (Bercot, 2017) for running persistent services, including the RStudio ${ }^{\circledR}$ server. This system provides a convenient way for downstream Dockerfile developers to add additional persistent services (such as an ssh server) to a single container, or additional start-up or shutdown scripts that should be run when a container starts up or shuts down. The rstudio image uses such a start-up script to configure user settings such as login password and permissions through environmental variables at run time.

The tidyverse image contains all required and suggested dependencies of the commonly-used tidyverse and devtools R packages, including external database libraries (e.g., MariaDB and PostgreSQL). Users should consult the package Dockerfiles or installed. packages() list directly for a complete list of installed packages. The verse library adds commonly-used dependencies, notably a large but not comprehensive LaTeX environment and Java development libraries. Previously, the Rocker project provided the image hadleyverse which has since been divided into tidyverse and verse based on community input.

Table 3: Available tags in the rocker-versioned stack.

\begin{tabular}{|c|c|c|c|c|}
\hline tag & apt repos & MRAN date & Build frequency & images with tag \\
\hline devel & stretch & current date & nightly & $\begin{array}{l}\text { r-ver, rstudio, tidyverse, } \\
\text { verse, geospatial }\end{array}$ \\
\hline latest & stretch & current date & nightly & $\begin{array}{l}r \text {-ver, rstudio, tidyverse, } \\
\text { verse, geospatial }\end{array}$ \\
\hline 3.4 .2 & stretch & current date & monthly & $\begin{array}{l}\text { r-ver, rstudio, tidyverse, } \\
\text { verse, geospatial }\end{array}$ \\
\hline 3.4 .1 & stretch & $2017-09-28$ & monthly & $\begin{array}{l}r \text {-ver, rstudio, tidyverse, } \\
\text { verse, geospatial }\end{array}$ \\
\hline 3.4 .0 & stretch & 2017-06-30 & monthly & $\begin{array}{l}\text { r-ver, rstudio, tidyverse, } \\
\text { verse, geospatial }\end{array}$ \\
\hline 3.3 .3 & jessie & 2017-04-21 & monthly & $\begin{array}{l}r \text {-ver, rstudio, tidyverse, } \\
\text { verse, geospatial }\end{array}$ \\
\hline 3.3.2 & jessie & 2017-03-06 & monthly & $\begin{array}{l}r \text {-ver, rstudio, tidyverse, } \\
\text { verse, geospatial }\end{array}$ \\
\hline 3.3 .1 & jessie & 2016-10-31 & monthly & $\begin{array}{l}r \text {-ver, rstudio, tidyverse, } \\
\text { verse, geospatial }\end{array}$ \\
\hline 3.3 .0 & jessie & 2016-06-21 & monthly & $r$-ver \\
\hline 3.2 .0 & jessie & 2015-06-18 & monthly & $r$-ver \\
\hline 3.1 .0 & jessie & 2014-09-17 & monthly & $r$-ver \\
\hline
\end{tabular}

Several images in the rocker-versioned stack can be customized on build when built locally (rather than pulling prebuilt images from Docker Hub) by using the --build-arg option of docker build. In the $r$-ver image, users can set R_VERSION and BUILD_DATE (MRAN default snapshot). In the rstudio image users can set RSTUDIO_VERSION (otherwise defaults to the most recent version), and the PANDOC_TEMPLATES_VERSION.

This stack also makes use of Docker metadata labels defined by http://schema-label.org, indicating image license (GPL-2.0), vcs-url (GitHub repository), and vendor (Rocker Project). These metadata can be altered or extended in downstream images.

\section{Conclusions}

Over the past several years, Docker has seen immense adoption across industry and academia. The Open Container initiative (The Linux Foundation: Projects, 2017) now provides an open standard 
that has further extended this container approach to research environments through projects such as Singularity (Lawrence Berkeley National Laboratories, 2017), allowing users to deploy containerized environments such as Rocker on machines where they do not have root access, such as clusters or private servers. Containerization promises to solve numerous challenges such as portability and replicability in research computing, which often relies on complex and heterogeneous software stacks (Boettiger, 2015). Yet implementing such environments in containers is not a trivial task, and not all implementations provide the same usability, portability or reproducibility. Here we have detailed the approach taken by the Rocker project in creating and maintaining these environments through an open and community-driven process. This structure of the Rocker project has evolved over three years of operation while drawing in an ever-widening base of academic researchers, university instructors and industry users. We believe this overview will be instructive not only to users and developers interested in the Rocker project, but as a model for similar efforts around other environments or domains.

\section{Bibliography}

L. Bercot. S6: Skarnet.org's Small and Secure Supervision Software Suite, 2017. URL https://skarnet. org/software/s6/. [p534]

C. Boettiger. An Introduction to Docker for Reproducible Research, with Examples from the R Environment. ACM SIGOPS Operating Systems Review, 49(1):71-79, 2015. URL https: //doi .org/ 10.1145/2723872.2723882. [p527, 530,535]

C. Boettiger and D. Eddelbuettel. Introducing Rocker: Docker for R, 2014. URL https://ropensci. org/blog/blog/2014/10/23/introducing-rocker. [p527]

M. Cetinkaya-Rundel and C. W. Rundel. Infrastructure and tools for teaching computing throughout the statistical curriculum. PeerJ Preprints, 5:e3181v1, 2017. ISSN 2167-9843. URL https://doi .org/ 10.7287/peerj.preprints.3181v1. [p528]

D. Clark, A. Culich, B. Hamlin, and R. Lovett. BCE: Berkeley's Common Scientific Compute Environment for Research and Education. Proceedings of the 13th Python in Science Conference (SciPy 2014), pages 1-8, 2014. [p527]

G. Csárdi. Rhub: Connect to 'R-Hub', from 'R', 2017. URL https://github.com/r-hub/rhub. R package version 1.0.1. [p530]

Debian Project. Apt-preferences overview, 2017. URL https://wiki.debian.org/AptPreferences. [p531]

Docker. What is Docker?, 2015. URL https://www. docker. com/what-docker. [p527, 529]

D. Eddelbuettel. Sanitizers, 2014. URL http://dirk.eddelbuettel.com/code/sanitizers.html. [p529, 532]

Lawrence Berkeley National Laboratories. Singularity, 2017. URL http://singularity.1bl.gov/. [p528, 535]

B. Ludaescher, K. Chard, M. Turk, V. Stodden, and N. Gaffney. The Whole Tale: Merging science and cyberinfrastructure pathways, 2017. URL https://wholetale.org/. [p530]

M. Plummer. JAGS: A program for analysis of Bayesian graphical models using Gibbs sampling, 2017. URL http://mcmc-jags. sourceforge. net/. Version 4.3.0. [p533]

Revolution Analytics. Microsoft R Application Network, 2017. URL https://mran. microsoft. com. [p530, 533]

C. A. Stewart, G. Turner, M. Vaughn, N. I. Gaffney, T. M. Cockerill, I. Foster, D. Hancock, N. Merchant, E. Skidmore, D. Stanzione, J. Taylor, and S. Tuecke. Jetstream: A Self-Provisioned, Scalable Science and Engineering Cloud Environment. In Proceedings of the 2015 XSEDE Conference on Scientific Advancements Enabled by Enhanced Cyberinfrastructure - XSEDE '15, pages 1-8, New York, New York, USA, 2015. ACM Press. ISBN 9781450337205. URL https://doi .org/10.1145/2792745.2792774. [p527]

The Linux Foundation: Projects. The Open Container Initiative, 2017. URL https://www. opencontainers.org/. [p534]

UC Berkeley. Curriculum Overview I Division of Data Sciences, 2017. URL http://data. berkeley . edu/education/curriculum-overview. [p528] 
K. Ushey, J. McPherson, J. Cheng, A. Atkins, and J. Allaire. Packrat: A Dependency Management System for Projects and Their R Package Dependencies, 2016. URL https://CRAN. R-project.org/package= packrat. R package version 0.4.8-1. [p530]

H. Wickham. Tidyverse: Easily Install and Load 'Tidyverse' Packages, 2017. URL https://CRAN. Rproject. org/package=tidyverse. $\mathrm{R}$ package version 1.1.1. [p533]

Carl Boettiger

UC Berkeley

ESPM Department, University of California,

130 Mulford Hall Berkeley, CA 94720-3114, USA

ORCiD 0000-0002-1642-628X

cboettig@berkeley. edu

Dirk Eddelbuettel

Debian and R Projects; Ketchum Trading

Chicago, IL, USA

ORCiD 0000-0001-6419-907X

edd@debian .org 